Article

\title{
Development of Monoclonal Antibodies and Antigen-Capture ELISA for Human Parechovirus Type 3
}

\author{
Keiko Goto ${ }^{1,2}$, Yutaro Yamaoka ${ }^{1,3}$, Hajera Khatun ${ }^{1}$, Kei Miyakawa ${ }^{1}$, Mayuko Nishi ${ }^{1}$, \\ Noriko Nagata ${ }^{2}$, Toshikazu Yanaoka ${ }^{2}$, Hirokazu Kimura ${ }^{4}$ and Akihide Ryo ${ }^{1, *}$ \\ 1 Department of Microbiology, Yokohama City University School of Medicine, Kanagawa 236-0004, Japan; \\ t176023b@yokohama-cu.ac.jp (K.G.); yutaro.yamaoka@gmail.com (Y.Y.); hkhatun1@gmail.com (H.K.); \\ keim@yokohama-cu.ac.jp (K.M.); mnishi@yokohama-cu.ac.jp (M.N.) \\ 2 Ibaraki Prefectural Institute of Public Health, Ibaraki 310-0852, Japan; eiken1@pref.ibaraki.lg.jp (N.N.); \\ eiken4@pref.ibaraki.lg.jp (T.Y.) \\ 3 Life Science Laboratory, Technology and Development Division, Kanto Chemical Co., Inc., \\ Kanagawa 259-1146, Japan \\ 4 Department of Health Science, Gunma Paz University Graduate School, Gunma 370-0006, Japan; \\ h-kimura@paz.ac.jp \\ * Correspondence: aryo@yokohama-cu.ac.jp; Tel.: +81-45-787-2602
}

Received: 25 August 2020; Accepted: 17 September 2020; Published: 19 September 2020

\begin{abstract}
Human parechovirus type $3(\mathrm{HPeV} 3)$ is an etiologic agent of respiratory diseases, meningitis, and sepsis-like illness in both infants and adults. Monoclonal antibodies (mAbs) can be a promising diagnostic tool for antigenic diseases such as virus infection, as they offer a high specificity toward a specific viral antigen. However, to date, there is no specific $\mathrm{mAb}$ available for the diagnosis of HPeV3 infection. In this study, we developed and characterized mAbs specific for $\mathrm{HPeV} 3$ capsid protein VP0. We used cell-free, wheat germ-synthesized viral VP0 protein for immunizing BALB/c mice to generate hybridomas. From the resultant hybridoma clones, we selected nine clones producing $\mathrm{mAbs}$ reactive to the HPeV3-VP0 antigen, based on enzyme-linked immunosorbent assay (ELISA). Epitope mapping showed that these mAbs recognized three distinct domains in HPeV3 VP0. Six mAbs recognized $\mathrm{HPeV} 3$ specifically and the other three mAbs showed cross-reactivity with other HPeVs. Using the HPeV3-specific mAbs, we then developed an ELISA for viral antigen detection that could be reliably used for laboratory diagnosis of HPeV3. This ELISA system exhibited no cross-reactivity with other related viruses. Our newly developed mAbs would, thus, provide a useful set of tools for future research and ensure $\mathrm{HPeV} 3$-specific diagnosis.
\end{abstract}

Keywords: human parechovirus; monoclonal antibodies; ELISA; VP0

\section{Introduction}

Human parechoviruses (HPeVs) belong to the Parechovirus genus of the Picornaviridae family [1,2]. The two serotypes ( $\mathrm{HPeV} 1$ and $\mathrm{HPeV} 2)$ of parechoviruses were initially isolated in 1956 from children with diarrhea, and were assigned to the Enterovirus genus. However, it became evident that they were genetically distinct from the enteroviruses, and reclassified as the Parechovirus genus in 1999. At present, $19 \mathrm{HPeVs}$ are reported and categorized, based on the nucleic acid sequences of the VP1 gene, not on the classification as enteroviruses serotype. HPeV3 was first identified in Japan. HPeV3 was isolated from a stool sample provided by a 1-year-old infant who was experiencing fever, gastritis like symptoms, and transient lower extremity paralysis [3]. HPeV types 1 to 8 are the common identified strains; among them, $\mathrm{HPeV}$ type 1, 3, and 6 account for the majority of infectious strains 
worldwide [4]. Infection with HPeVs is associated with a broad spectrum of clinical manifestations, ranging from respiratory symptoms and mild gastrointestinal illness to sepsis-like diseases, meningitis, and encephalitis in children [5]. While most HPeVs cause mild symptoms in children between 1 and 5 years of age, human parechovirus 3 (HPeV3) is clinically the most important genotype, owing to its association to severe diseases in younger infants under 3 months of age [6-8]. HPeV3 infection in infants can trigger a sepsis-like dysregulated host response involving the central nervous system [9-12]. In cases of acute meningitis or encephalitis, patients might develop abnormal white matter lesions and neurological sequelae, and even death might occur [13-17]. Apnea can occur in children regardless of encephalitis [17]. HPeV3 is known to cause myalgia and myositis in adult patients and a similar pattern is also sporadically seen among pediatric patients. [18,19]. An epidemic of HPeV3 occurs every 3 to 4 years in Japan. As respiratory disease or meningitis cases due to $\mathrm{HPeV} 3$ are not subject to notifiable disease surveillance in Japan, the actual number of the patients is not known [1,6,20-23]. The appropriate diagnosis tool for $\mathrm{HPeV} 3$ detection might be able to rule out infectious etiology and avoid unnecessary antibiotics use, which is a given because HPeV3 leads to septic shock-like symptoms. For these reasons, the establishment of a method to detect $\mathrm{HPeV} 3$ plays a vital role in healthcare fields. Importantly, $\mathrm{HPeV}^{\prime}$ 's epidemic cycle occurs in summer time and is concurrent with enteroviral infection. Thus, it is essential to develop a detection method that does not cross-react with Enterovirus.

HPeV3 contains a small, non-enveloped, single-stranded positive-sense RNA genome of approximately 7.3-kb nucleotides [24,25]. The HPeV3 virion is composed of 60 copies of three structural proteins (VP0, VP1, and VP3) that fit together to form a 28-nm-diameter icosahedral shell around the viral genome [26]. The genome encodes a single polyprotein that, during infection, is subsequently cleaved into all essential capsid components and non-structural proteins [24]. VP0 is an important protein for stabilizing the surface of the viral capsid, and the assembly of $\mathrm{HPeV}$ is controlled by multiple interactions of the genome with the capsid, through conserved amino acids in VP1 and VP3 [25].

Although RT-PCR-based diagnostic tests targeting $5^{\prime}$-UTR of the HPeV3 genome were developed for $\mathrm{HPeV} 3$ detection in clinical samples, there is currently no diagnostic method for detecting the viral antigens. Recently, Chen et al. generated polyclonal antibodies for HPeV3 VP0, and proposed an immunofluorescence-based diagnostic assay [27]. However, this method requires virus isolation by cell culture and takes several weeks for the identification of viral genotype/serotype. Abed et al. developed a serological enzyme-linked immunosorbent assay (ELISA), using a synthetic peptide from the VP0 protein of HPeVs [28]. Although it can provide a definitive diagnosis, serological test requires paired serum samples from acute and recovery phases, which makes it difficult to diagnose immediately as a point of care testing (POCT). To develop a rapid and effective diagnostic strategy, there is an urgent need to produce highly specific monoclonal antibodies (mAbs) toward $\mathrm{HPeV} 3$ antigens.

In this study, we sought to generate mAbs specific to the capsid protein VP0 of $\mathrm{HPeV}$. We prepared the viral VP0 antigen using the wheat germ cell-free system, which has the advantage of producing properly folded functional proteins $[29,30]$, to immunize mice. As a result, we obtained nine $\mathrm{mAb}$ clones for characterization, and thereafter, generated an ELISA system that is specifically able to detect the HPeV3 VP0 antigens.

\section{Materials and Methods}

\subsection{Construction of Expression Vector}

Complementary DNA encoding HPeV3-VP0 (GenBank No. AB084913) was used to generate the expression vector for antigen production with the wheat germ cell-free system. The HPeV3-VP0 open reading frame was amplified by PCR, using the corresponding primer pairs. The amplified fragment was cloned into vector pEU-E01-His-TEV-MCS-N2 (CellFree Sciences, Yokohama, Japan), using restriction enzymes XhoI and SpeI. 


\subsection{Cell-Free Protein Synthesis}

In vitro wheat germ cell-free protein synthesis was carried out as previously described $[29,30]$. For cell-free protein synthesis, WEPRO7240H wheat extract (CellFree Sciences, Yokohama, Japan) was used in the bilayer translation reaction, as previously described. Synthesized proteins were confirmed by immunoblotting. The His-HPeV3-VP0 (full length, 1-298) protein was synthesized using a Proteomist XE robotic protein synthesizer (CellFree Sciences, Yokohama, Japan) for mouse immunization. The cell-free translation reaction mixture was separated into soluble and insoluble fractions by centrifugation at $18,000 \times g$ for $15 \mathrm{~min}$. The soluble fraction was mixed with Ni-Sepharose High Performance beads (GE Healthcare, Waukesha, WI, USA) in the presence of $20 \mathrm{mM}$ imidazole. The beads were washed thrice with a washing buffer [20 mM Tris- $\mathrm{HCl}(\mathrm{pH} 7.5), 500 \mathrm{mM} \mathrm{NaCl}]$ containing $40 \mathrm{mM}$ imidazole. His-HPeV3-VP0 was then eluted in another washing buffer containing $500 \mathrm{mM}$ imidazole. Amicon Ultra centrifugal filters (Millipore, Bedford, MA, USA) were used to concentrate the purified His-HPeV3-VP0. Protein concentration was determined using the Bradford method, with bovine serum albumin (BSA) as a protein standard.

\subsection{Monoclonal Antibody Production}

Immunization of BALB/c mice and generation of anti-HPeV3-VP0 mAb-producing hybridomas were carried out as previously described $[29,30]$. Briefly, His-tagged full-length HPeV3-VP0 protein was injected into the footpad of the BALB/c mice, using keyhole limpet hemocyanin as an adjuvant. Four weeks later, the spleen cells were isolated and fused to the myeloma cell line, SP2/O, using polyethylene glycol 1500 (PEG 1500). Monoclonal antibodies in the hybridoma culture supernatant were tested using ELISA with His-tagged recombinant HPeV3-VP0 protein. Isotype determination was performed using Isostrip mouse monoclonal antibody isotyping kit, following the manufacturer's instructions (Roche Diagnostics, Basel, Switzerland).

\subsection{Cell and Virus Culture}

Vero cells were grown in DMEM containing 10\% FBS. HPeV3 was provided by Dr. Masaki Takahashi (Iwate Prefectural Institute of Public Health). HPeV3 was propagated in Vero cells and quantified by qRT-PCR. The sequence information was as follows: 5'-GTAACASWWGCCTCTGGGS CCAAAAG-3' (Forward primer), 5'-GGCCCCWGRTCAGATCCAYAGT-3' (Reverse primer), and 5'-VIC-CCTRYGGGTACCTYCWGGGCATCCTTC-BHQ-3' (Probe).

\subsection{Immunoblotting}

Wheat germ-synthesized recombinant His-tagged HPeV3-VP0 proteins were separated by $10 \%$ SDS-PAGE in running buffer ( $250 \mathrm{mM}$ glycine, $25 \mathrm{mM}$ Tris, $0.1 \%$ SDS). The separated proteins were transferred to a PVDF membrane (Millipore). The membranes were washed with blotting buffer TBST (TBS containing 0.05\%-Tween 20), and then blocked for $1 \mathrm{~h}$ at room temperature in $5 \%$ non-fat powdered milk in TBST. Thereafter, the membranes were incubated overnight with generated hybridoma supernatant (1:50 dilution in TBST) at $4{ }^{\circ} \mathrm{C}$. Next, after washing thrice in TBST, the membranes were incubated for $1 \mathrm{~h}$ at room temperature with anti-mouse IgG-HRP secondary antibody (1:10000 dilution in TBST). Finally, after washing thrice in TBST, the target protein was detected with the Immobilon Western Chemiluminescence detection system (GE Healthcare) using Fluor Chem FC2 (Alpha Innotech Corp. Tokyo, Japan).

\subsection{Epitope Mapping}

For epitope mapping, we prepared deletion mutants of HPeV3-VP0 using PCR mutagenesis with template vector $\mathrm{pEU}-\mathrm{His}-\mathrm{HPeV} 3-\mathrm{VP} 0$, followed by wheat germ cell-free protein synthesis. The proteins were analyzed by immunoblotting using our generated mAbs. 


\subsection{Multiple Sequence Alignment}

To examine the amino acid variability among the VP0 proteins of other $\mathrm{HPeV}$ genotypes, VP0 sequences for HPeV1-8, 14, and 17-19 were accessed from the GenBank and aligned with the HPeV3-VP0 sequence using the MEGA software.

\subsection{Affinity Measurement of $m A b s$}

$\mathrm{K}_{\mathrm{D}}$ values were determined by Bio-Layer interferometry (BLI) using Octet RED96 (ForteBio, USA). Anti-mouse IgG Capture biosensor tips (AMC, ForteBio) were loaded with $20 \mu \mathrm{g} / \mathrm{mL}$ of $\mathrm{mAb} \# 8$ and \#39 for $5 \mathrm{~min}$, in PBS containing 0.1\% BSA and 0.01\% Tween 20 . The association of recombinant VP0 at concentrations of 50, 25, 12, and $5 \mathrm{nM}$ for \#8 $\mathrm{mAb}$, and 14, 7, 3.5, and $1.75 \mathrm{nM}$ for \#39 $\mathrm{mAb}$, was measured for $5 \mathrm{~min}$, followed by a 10-min-long dissociation phase. All measurements were corrected for baseline drift by subtracting a reference well. The operating temperature was maintained at $30^{\circ} \mathrm{C}$. Data were analyzed using a 1:1 binding model with global fitting algorithms in the ForteBio data analysis software.

\subsection{Selection of the Optimal Pair of mAbs for Sandwich ELISA}

Each $\mathrm{mAb}$ was diluted in $50 \mathrm{mM}$ of carbonate buffer ( $\mathrm{pH}$ 9.6) to a concentration of $10 \mu \mathrm{g} / \mathrm{mL}$, and then added to an ELISA plate (AGC TECHNO GLASS, Shizuoka, Japan). To immobilize the antibodies, the plate was incubated overnight at $4{ }^{\circ} \mathrm{C}$. Wells were blocked with PBS containing $2 \%$ $(\mathrm{w} / \mathrm{v})$ skim milk for $1 \mathrm{~h}$ at room temperature (RT). After three washes with PBS containing $0.05 \%(\mathrm{v} / \mathrm{v})$ Tween-20 (PBS-T), $100 \mu \mathrm{L}$ of antigen protein $(8 \mathrm{ng} / \mathrm{mL}$ ) diluted with PBS-T or blank (PBS-T alone) was added, and the mixture was incubated for $60 \mathrm{~min}$ at RT. After three washes with PBS-T, $100 \mu \mathrm{L}$ of each $\mathrm{mAb}$, conjugated with horseradish peroxidase (HRP), was added into each well and incubated for $60 \mathrm{~min}$ at RT. Antibody labeling was performed using the Peroxidase Labeling Kit-NH2 (Dojindo Laboratories, Kumamoto, Japan). After three washes with PBS-T, $100 \mu \mathrm{L}$ of ABTS substrate solution (Kirkegaard and Perry Laboratories, Washington, DC, USA) was added and the mixture was incubated for $30 \mathrm{~min}$ at RT. Absorbance at 405/490 nm was measured on GloMax Discover System (Promega), and the signal-to-noise ratio $(\mathrm{S} / \mathrm{N})$ was calculated.

\section{Results}

\subsection{Generation of $m A$ bs to Target HPeV3-VPO Protein}

For generation of mAbs, we produced N-terminal His-tagged full-length VP0 protein of HPeV3 as an antigen. As a result, HPeV3-VP0 protein was produced with high aqueous solubility (Figure 1a). The protein was subsequently purified using Ni-Sepharose beads, followed by elution with imidazole. BALB/c mice were then immunized with the purified protein. After 4 weeks of immunization, the mice splenocytes were isolated, fused with myeloma cells, and hybridomas were produced. As a result, 48 stable hybridomas were generated and designated as \#1 to \#48. Among these 48 clones, nine were selected (\#3,\#6, \#8,\#12,\#27,\#30,\#34,\#39, and \#41), based on the reactivity in ELISA to the target antigens VP0 proteins derived from $\mathrm{HPeV} 1$ and $\mathrm{HPeV} 3$ (Figure 1b). Isotype analysis revealed that \#3 mAb belongs to IgG1, kappa isotype, \#6 and \#12 mAbs belong to IgG2a, kappa isotype, while the others belongs to IgG2b, kappa isotype (Figure 1c). 
a

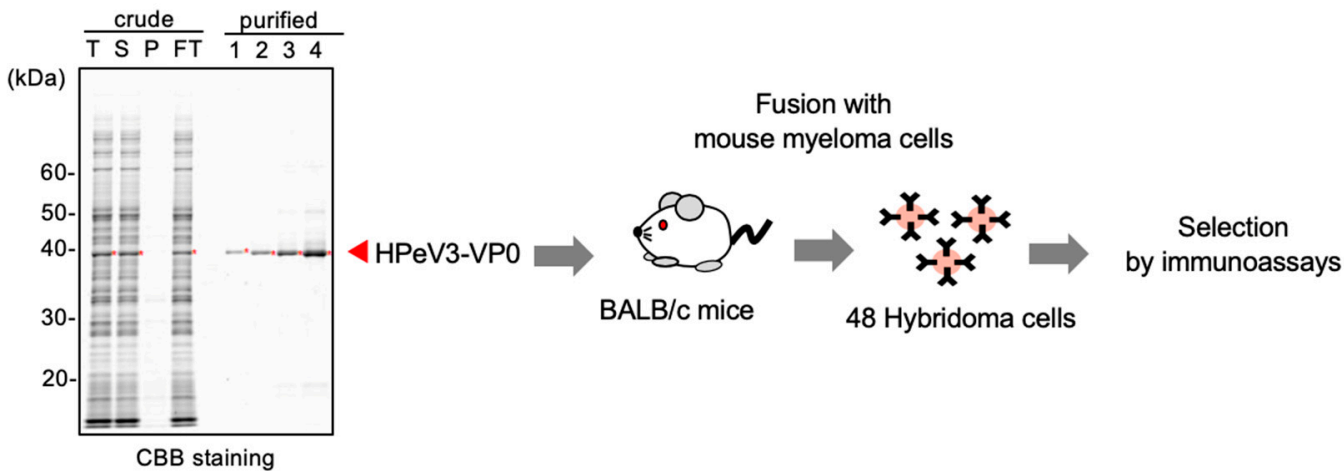

b

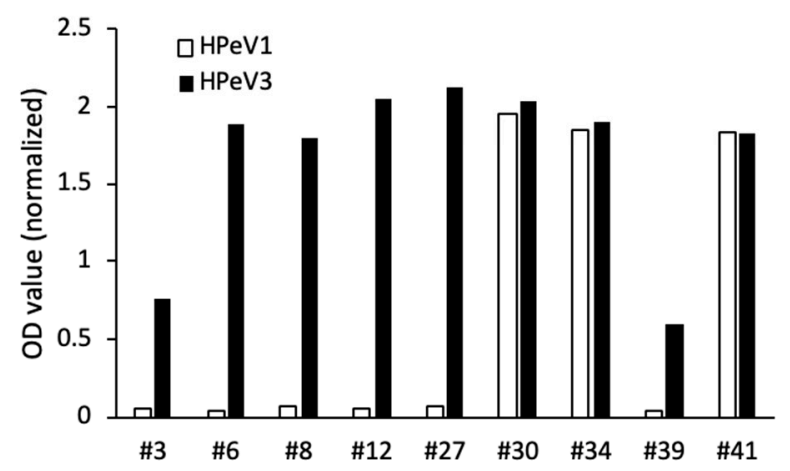

c

\begin{tabular}{ccc}
$\begin{array}{c}\text { mAb } \\
\text { clone No }\end{array}$ & Isotype & $\begin{array}{c}\text { Light } \\
\text { Chain }\end{array}$ \\
\hline$\# 3$ & IgG1 & $\mathrm{k}$ \\
$\# 6$ & IgG2a & $\mathrm{k}$ \\
$\# 8$ & IgG2b & $\mathrm{k}$ \\
$\# 12$ & IgG2a & $\mathrm{k}$ \\
$\# 27$ & IgG2b & $\mathrm{k}$ \\
$\# 30$ & IgG2b & $\mathrm{k}$ \\
$\# 34$ & IgG2b & $\mathrm{k}$ \\
$\# 39$ & IgG2b & $\mathrm{k}$ \\
$\# 41$ & IgG2b & $\mathrm{k}$
\end{tabular}

Figure 1. Production of anti-HPeV3 mAbs using wheat germ cell-free synthesized VP0 antigen. (a) Schematic representation of hybridoma cell production to generate anti-HPeV3 mAbs using the recombinant Histidine-tagged HPeV3-VP0 (His-VP0) protein produced by the wheat germ cell-free system, and then purified using nickel-chelated Sepharose beads. Each protein fraction was analyzed by SDS-PAGE and visualized by CBB staining. Red dots and arrow indicate the target protein. T-total fraction; S-supernatant; P-precipitate; FT-flow-through; and purified fractions 1-4. Purified His-HPeV3-VP0 antigen was injected into BALB/c mice. After 4 weeks, the spleen cells of immunized mice were fused with myeloma cells and 48 hybridoma clones were established. (b) Out of these 48 clones, nine exhibited relatively high reactivity to antigen proteins, as revealed by ELISA. We used HPeV1 and $\mathrm{HPeV} 3 \mathrm{VP0}$ proteins as antigens. (c) Isotyping of selected mAbs-producing hybridoma clones.

\subsection{Epitope Mapping of Anti-HPeV3-VPO $m A b$}

To demonstrate the antibody-binding sites within the antigen, we next performed epitope mapping. For epitope mapping, we produced five deletion mutants of VP0 and performed immunoblotting analysis. We found that our newly developed $\mathrm{mAbs}$ recognized three distinct domains in HPeV3 VP0: \#30 and \#34 mAbs bind to 68-121 amino acid (aa), \#3, \#8, and \#27 mAbs bind to 127-172 aa, and remaining four $\mathrm{mAbs}(\# 6, \# 12, \# 39$, and \#41) bind to 225-289 aa within C-terminal region of the VP0 protein of HPeV3 (Figure 2a). We further created deletion mutants for a more precise epitope determination for these mAbs, and found that \#30 and \#34 mAbs bind to 82-95 aa, \#3, \#8, and \#27 $\mathrm{mAbs}$ bind to 133-159 aa, and \#6, \#12,\#39, and \#41 mAbs bind to 275-289 aa (Figure 2b). We next examined whether the antigenic epitopes were located on the surface of HPeV3-VP0. The UCSF Chimera software revealed that, except for \#30 and \#34, the binding regions of all $\mathrm{mAbs}$ were located on the molecular surface of VP0 protein (Figure 2c). The binding region of mAbs \#30 and \#34 was relatively conserved among the analyzed $\mathrm{HPeVs}$ (Figure 2d). 
a

\begin{tabular}{c|ccccccccc} 
& $\# 3$ & $\# 6$ & $\# 8$ & $\# 12$ & $\# 27$ & $\# 30$ & $\# 34$ & $\# 39$ & $\# 41$ \\
\hline $1-67$ & + & + & + & + & + & + & + & + & + \\
$68-121$ & + & + & + & + & + & - & - & + & + \\
$122-172$ & - & + & - & + & - & + & + & + & + \\
$173-224$ & + & + & + & + & + & + & + & + & + \\
$225-289$ & + & - & + & - & + & + & + & - & - \\
Epitope (aa) & $122-172$ & $225-289$ & $122-172$ & $225-289$ & $122-172$ & $68-121$ & $68-121$ & $225-289$ & $225-289$
\end{tabular}

b

\begin{tabular}{c|cc} 
& $\# 30$ & $\# 34$ \\
\hline $68-81$ & + & + \\
$82-95$ & - & - \\
$96-109$ & + & + \\
$110-121$ & + & + \\
Epitope (aa) & \multicolumn{2}{|c}{$82-95$}
\end{tabular}

\begin{tabular}{c|ccc} 
& $\# 3$ & $\# 8$ & $\# 27$ \\
\hline $122-132$ & + & + & + \\
$133-145$ & - & - & - \\
$146-159$ & - & - & - \\
$160-172$ & + & + & + \\
Epitope(aa) & \multicolumn{3}{|c}{$133-159$}
\end{tabular}

\begin{tabular}{c|cccc} 
& $\# 6$ & $\# 12$ & $\# 39$ & $\# 41$ \\
\hline $225-240$ & + & + & + & + \\
$241-258$ & + & + & + & + \\
$259-275$ & + & + & + & + \\
$275-289$ & - & - & - & - \\
Epitope (aa) & & $275-289$ &
\end{tabular}

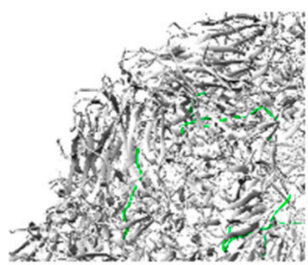

Epitope 82-95

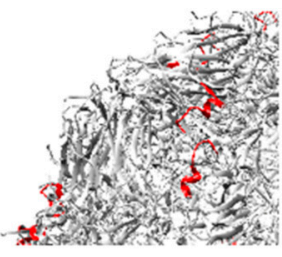

Epitope 133-159

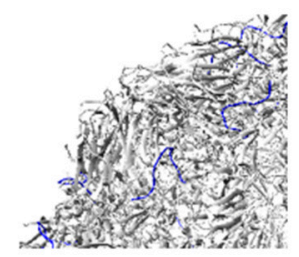

Epitope 275-289

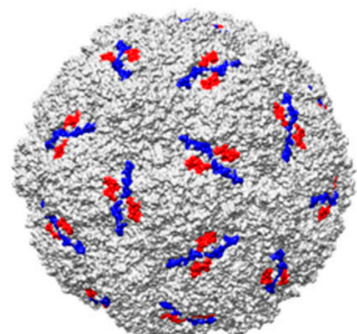

d

Amino acid number

\begin{tabular}{|c|c|c|c|}
\hline Name of strains & $82-95$ & $133-159$ & 275-289 \\
\hline (AB084913) & QHPSAPTIPFTPDF & QYEITTVELPKSFWDDTRKPAYGQAKY & QNPRPYGEDVDIYDN \\
\hline HPeV1_(L02971) & $. M . S$ & G.Q. H ..... . HQD ...SR & $\ldots V F A Q \ldots N$ \\
\hline 5695$)$ & $\mathrm{L}$ & GHQ. DH.N. VD . EQ ..... . . & $\ldots V \ldots Q N \ldots T$ \\
\hline HPeV4_(DQ315670) & . . M. & $. H L . V D \ldots . . . H A \ldots N . N \ldots S R$ & $\ldots . \mathrm{V}, \mathrm{NTQ} \ldots$ \\
\hline (AF055846) & . M & . HL . ND . . . A . KNS . . . SR & $\ldots \mathrm{T} . \mathrm{DT} \ldots \mathrm{N}$ \\
\hline HPeV6_(AB252582) & $M$ & SDK.MH....A. . NEQ... & . NKQ.N. \\
\hline HPeV7_(EU556224) & & $R H \ldots A Y . G \ldots N G \ldots T E \ldots M \ldots H \ldots S R$ & $\ldots \mathrm{T} \ldots \mathrm{A}$. \\
\hline 5) & A & $S H Q . D Y \ldots A . \ldots A E K M \ldots F$. & $V . Q E . N$. \\
\hline HPeV14_(MG571810) & & HQLLNI . . RG . . NE . . F . . S . & $\ldots A \ldots V \ldots H$ \\
\hline 21) & & . HQ. MY .N. . SG. & $\ldots \mathrm{N} . \mathrm{E} . \mathrm{S}$ \\
\hline HPeV18_(KT879915) & & . HQ . & $\ldots N . E$. \\
\hline HPeV19_(KT879920) & $. M . M$. & EKLMDIG. TA. . N . N ....SR & $\ldots A F S \ldots \ldots$ \\
\hline
\end{tabular}

Figure 2. Epitope mapping of mAbs. (a,b) Deletion mutants of VP0 were produced by wheat germ cell-free protein synthesis. Reactivity of each $\mathrm{mAb}$ to the deletion mutants was evaluated by immunoblotting. + and - indicate positive and negative detection, respectively. (c) Position of epitopes in a structural model of HPeV3 VP0 (PDB ID. 6GV4). Epitope localizations of the various mAbs are highlighted in different colors. (d) Multiple alignment of sequences in HPeV VP0 proteins in the epitope region.

\subsection{Cross-Reactivity of Anti-HPeV3-VP0 mAbs}

We next investigated the specificity of our newly developed mAbs. For this, we created VP0 proteins encoded by the six different $\mathrm{HPeV}$ genotypes and VP4-VP2 proteins derived from Enteroviruses 71 and D68. Immunoblotting analysis showed that six mAbs (\#3, \#6, \#8, \#12, \#27, and \#39) react 
specifically with $\mathrm{HPeV} 3 \mathrm{VP0}$, while three mAbs (\#30, \#34, and \#41) showed some cross-reactivity to other HPeVs (Figure 3). No mAbs showed cross-reactivity to enterovirus VP4-VP2 proteins.

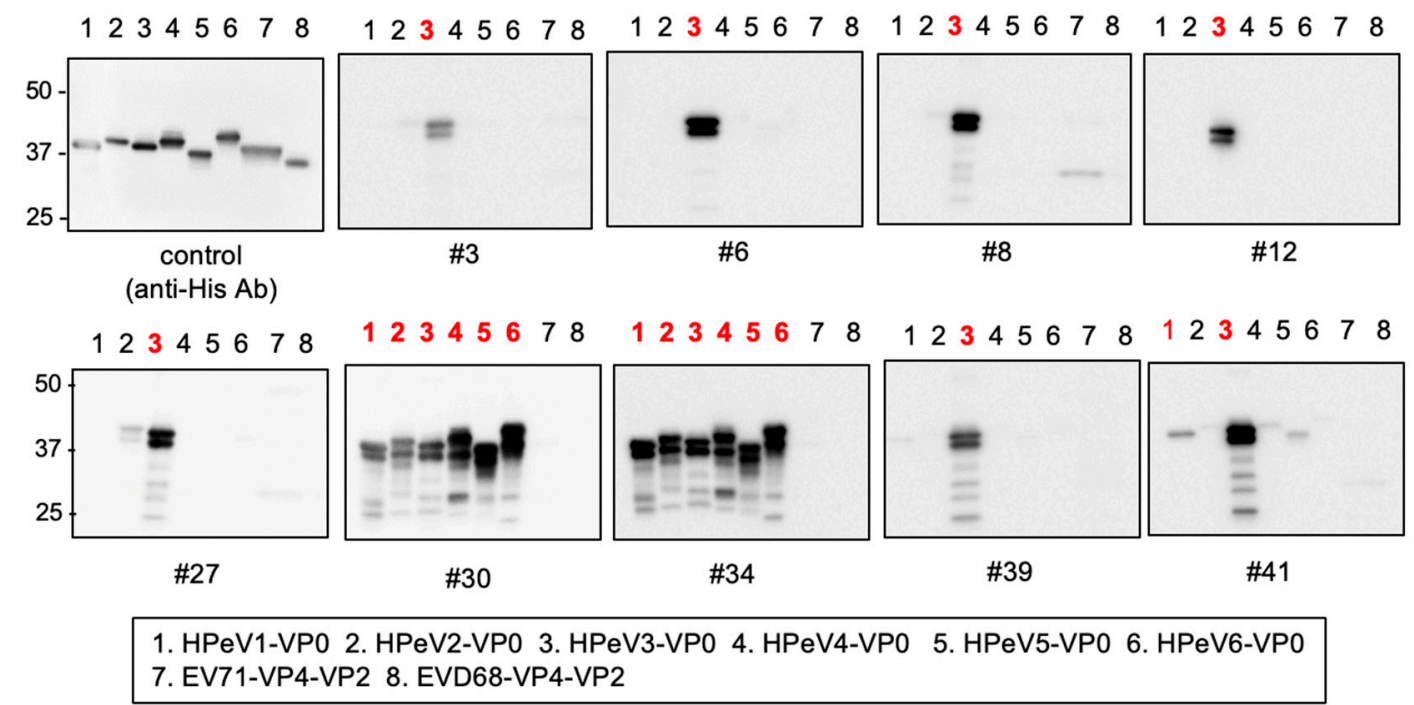

Figure 3. Cross-reactivity of anti- HPeV3-VP0 mAbs. Cross-reactivity of selected mAbs to related HPeVs. His-tagged VP0 proteins of HPeVs were synthesized by a wheat germ cell-free system and the reactivity of $\mathrm{mAbs}$ was then assessed by immunoblotting analysis. The numbers associated with lanes showing positive bands are highlighted in red.

\subsection{Development of Antigen-Capture ELISA for HPeV3 VPO}

Sandwich ELISA systems with highly specific matched antibody pairs are commonly used to detect and quantify viral antigens in immunoassay. Hence, we next determined the optimal pair of $m A b s$ for antigen-capture using ELISA, by evaluating all possible combinations of immobilized and labeled mAbs. Among the 36 possible pair combinations, only the \#8 and \#39 mAb pair represented a combination of antibodies specific for HPeV3-VP0 (Figure 4a). Therefore, we selected this combination for further analysis.

To characterize the equilibrium dissociation constant $\left(\mathrm{K}_{\mathrm{D}}\right)$ of the selected antibodies and the target VP0 antigen, we used the BLI Octet Assay System. $K_{D}$ for antibody-antigen binding in mAbs \#8 and $\# 39$ was calculated as $<1 \times 10^{-12}$ and $3.72 \times 10^{-10}$, respectively, suggesting that both $\mathrm{mAbs}$ showed high binding affinity to the HPeV3-VP0 (Figure $4 \mathrm{~b}$ ).

We next performed antigen-capture ELISA with recombinant VP0 protein and virions released into the cell-culture supernatant of the HPeV3-infected cells. Using the optimal antibody pair (\#8 and \#39 mAbs) identified above, we determined the detection threshold for antigen recognition by antigen-capture ELISA. Our results revealed that our system was highly sensitive to the recombinant antigen, capable of detecting the protein at a concentration of $3 \mathrm{ng} / \mathrm{mL}$ (Figure $4 \mathrm{c}$, left). In parallel, we investigated the detection limit of ELISA for the HPeV3 virion. This ELISA system could detect heat-treated $\mathrm{HPeV} 3$, but not non-heated virions, and its detection limit of the system was $1 \times 10^{9}$ copies/mL (Figure 4c, right). We also found that this ELISA system exhibited no cross-reactivity with enteroviruses (Figure $4 \mathrm{~d}$ ) 
a
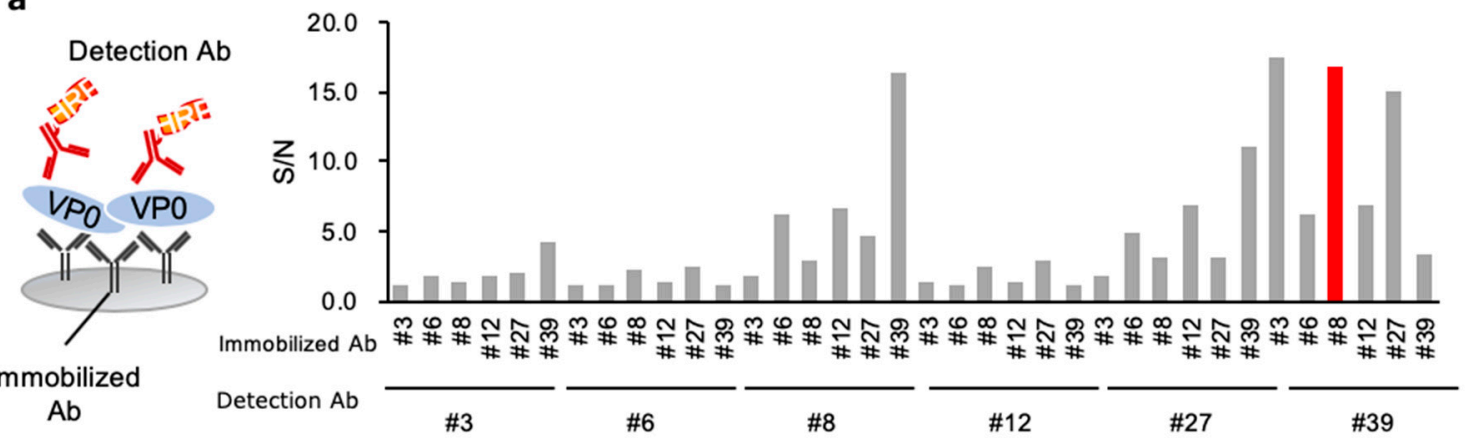

b
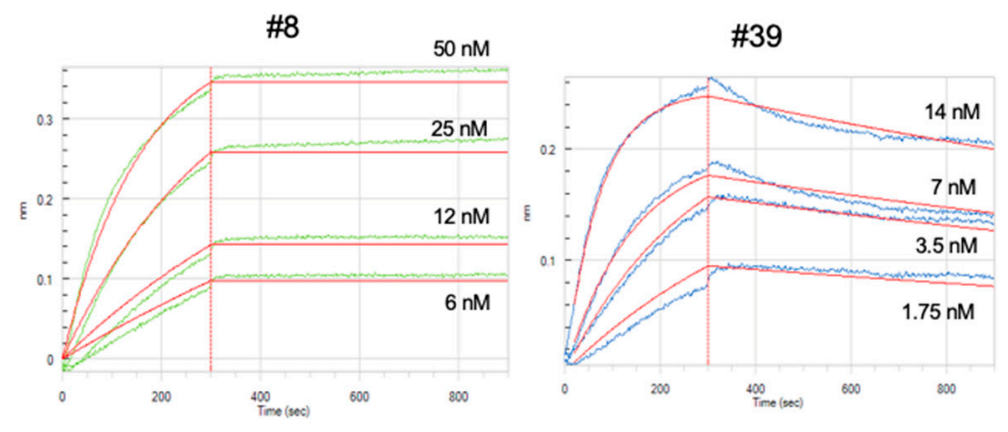

\begin{tabular}{c|cc}
$\mathrm{mAb}$ & $\# 8$ & $\# 39$ \\
\hline $\mathrm{K}_{\mathrm{D}}(\mathrm{M})$ & $<1.0 \times 10^{-12}$ & $3.7 \times 10^{-10}$ \\
$\mathrm{k}_{\mathrm{on}}(1 / \mathrm{Ms})$ & $1.3 \times 10^{5}$ & $9.6 \times 10^{5}$ \\
$\mathrm{k}_{\text {dis }}(1 / \mathrm{s})$ & $<1.0 \times 10^{-7}$ & $3.6 \times 10^{-4}$
\end{tabular}

C

d

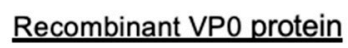

$\underline{\text { Viral particles }}$

Cross reactivity with related viruses
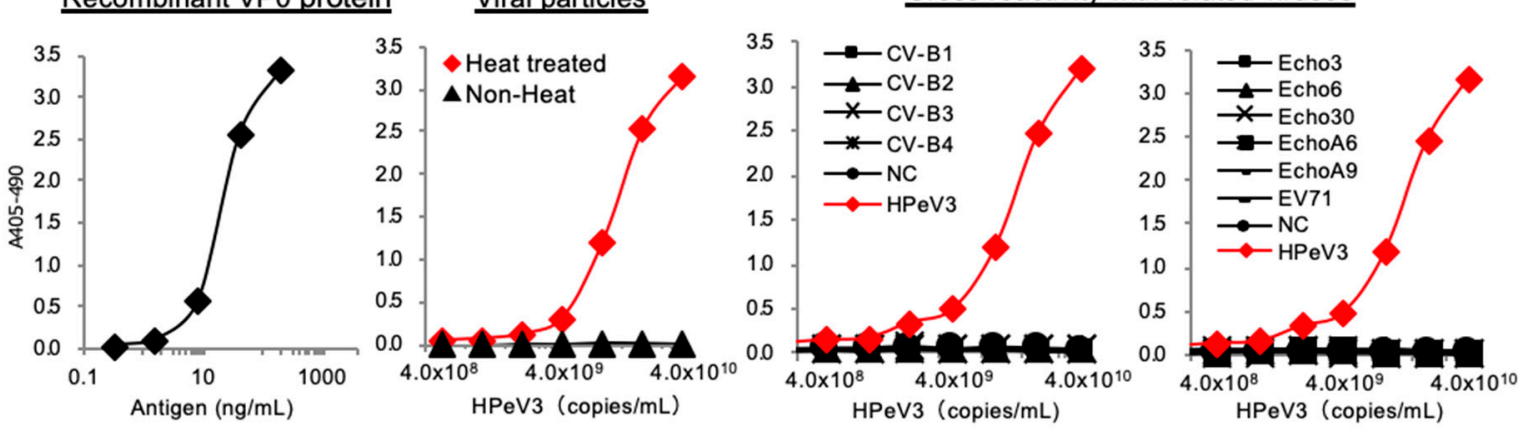

Figure 4. Development of antigen-capture ELISA for HPeV3 VP0. (a) Thirty-six mAb pairs were tested for sandwich ELISA. Signal-to-noise $(\mathrm{S} / \mathrm{N})$ ratio for antigen detection by each of the 36 combinations was calculated in the presence of $8 \mathrm{ng}$ antigen vs. blank. (b) $\mathrm{K}_{\mathrm{D}}$ value, $\mathrm{k}_{\mathrm{on}}$, and $\mathrm{k}_{\mathrm{dis}}$ of \#8 and \#38 mAbs. (c) Detection limit of ELISA for HPeV3 recombinant VP0 proteins (left) and viral particles (right).

(d) Cross-reactivity with related viruses of the HPeV3 ELISA system.

\section{Discussion}

$\mathrm{HPeV} 3$ is increasingly being highlighted as a potentially severe viral infection in neonates and young infants. Therefore, there is an urgent need to develop assays for early diagnosis of HPeV3 infection for reducing inappropriate antimicrobial use, unnecessary investigations, and prolonged hospitalization. It is also likely to lead to follow-up for potential complications in infants who are severely affected [1]. In this context, a real-time PCR-based molecular test to detect virus from patients was recently developed [31]. However, PCR tests are extremely sensitive and need extensive controls, whereas antigen detection by mAbs has the advantage of the relative ease of sample handling and the use of less stringent procedures. Specific mAbs could then be used to develop a rapid test such as ELISA. In this study, we sought to generate specific mAbs and develop an ELISA test for the detection of HPeV3 VP0 antigen. 
HPeV3 genome encodes for three structural proteins, namely VP0, VP3, and VP1, which assemble to create the virus particle [10]. Among these, VP0 was identified as an antigenic determinant and it might be relatively more useful for diagnostic purposes, due to a higher level of sequence conservation [32]. Furthermore, it possesses high immunogenicity [7]. Therefore, the selection of VP0 as an antigen is both practical and reasonable. In addition, the mAb quality was determined mostly by preparation of high-quality antigen. Here, we used a wheat germ, cell-free protein production system for synthesizing recombinant VP0 proteins, as this system produces properly folded, soluble, and biologically active native proteins similar to those expressed in mammalian cells [29,30,33].

In our current study, we newly produced nine different mAbs that recognized HPeV3-VP0 as an antigen. We then performed epitope mapping of our generated $\mathrm{mAbs}$, as identification of the epitope is a key step in the characterization of monoclonal antibodies [34]. Based on the epitope analysis, the mAbs were able to recognize three different areas of HPeV3-VP0 and specify 12-14 aa length epitopes within the HPeV3-VP0. Interestingly, two mAb clones (\#30 and \#34) exhibiting cross-reactivity to VP0 proteins of other HPeVs, bound a distinct epitope (82-95 aa), which partially overlapped with the recognition site for the polyclonal antibody (79-99 aa) created by Chen et al. [27]. Nevertheless, we obtained mAbs specific to HPeV3, which recognized sites 133-159 aa and the 275-280 aa site of VP0, which was not reported earlier. Moreover, we also developed an ELISA for detecting HPeV3 antigen using two of our newly generated HPeV3-specific mAbs (\#8 and \#39), as mAb-based ELISA is highly specific and sensitive towards viral antigen detection [35]. Our Octet assay suggested that both mAbs show a high binding affinity to the full-length HPeV3-VP0 recombinant protein. VP0 protein can be folded into the correct native structure and is likely to form the capsid-like structure via oligomerization. In this situation, \#8 mAb could bind various sites on polymerized VP0 proteins, as a result of an allosteric effect or "avidity", owing to which the $\mathrm{K}_{\mathrm{D}}$ value of \#8 mAb was calculated to be much lower than that of \#39 mAb.

Our newly developed ELISA system requires heat treatment of HPeV3 to detect VP0 antigen. Based on previous studies [7], the binding area of $\mathrm{mAb} \# 39$ was estimated to localize to the interface between VP0 and VP3. On the other hand, three-dimensional modeling of viral particles revealed that the antigen-recognizing sites of $\mathrm{mAbs} \# 8$ and \#39 in VP0 protein are proximally located. Moreover, a previous study showed that Glu285 (in the epitope region of \#39) and Ser28 (in the VP0 protein of $\mathrm{HPeV} 3$ ) bind together by a hydrogen bond [24]. Thus, a possible explanation for our observation is that heat treatment helps antigen retrieval for the interface between VP0 and VP3, resulting in efficient access of mAbs to the epitopes.

According to a previous study [36], an increase in antibody-binding capacity was exhibited when glycosylation of capsid protein was removed. Based on this finding, we carried out pretreatment by resecting the connection between O-linked and N-linked glycosylation of HPeV3. However, there was no obvious effect on antibody recognition in our sandwich ELISA assay (data not shown), indicating that glycosylation might not affect the antigenicity for $\mathrm{mAb}$ recognition.

The detection limits of our newly developed sandwich ELISA were $3 \mathrm{ng} / \mathrm{mL}$ for recombinant VP0 protein and $1 \times 10^{9}$ copies/mL for viral particles, respectively. Assuming that one viral particle contains a single copy of a viral genome, there are 60 copies of VP0 protein per viral particle. For a viral particle detection sensitivity of $1 \times 10^{9}$ copies/mL, the VP0 protein detection sensitivity is presumed to be $3 \mathrm{ng} / \mathrm{mL}$. Therefore, we conclude that the detection sensitivity for the recombinant VP0 protein and the virus particles was almost equivalent, suggesting the feasibility of our method for use in actual clinical settings.

Other than the ELISA method, several antigen-detecting tests using an antigen-antibody interaction are now available. For instance, the multi-array technology using electrochemiluminescence immunoassay (ECLIA) and chemiluminescent enzyme immunoassay (CLEIA) can provide several hundred times more sensitivities than the conventional ELISA method [37]. Another example is an influenza-testing kit using a highly sensitive immunochromatographic detection method based on silver amplification [38]. The limitation of sensitivity might be overcome by utilizing these sophisticated 
technologies combined with our monoclonal antibodies for HPeV3 VP0 antigen. Furthermore, we can potentially improve the detection sensitivity by altering the second antibody to recognize the poly-HRP complex [39] or by adding other antibodies, which can target VP1 or VP3 proteins.

In summary, we utilized the wheat germ cell-free protein production system to synthesize the $\mathrm{HPeV} 3 \mathrm{VP0}$ protein and produced mAbs that could specifically detect HPeV3 but not other HPeVs. We further explored the feasibility of these mAbs in terms of their utility in various immunological applications. To the best of our knowledge, the HPeV3 VP0 antibody as well as the ELISA-based viral detection system reported here is the first of its kind ever reported. With the implementation of more sophisticated applications, our newly developed mAbs could be useful for further development of diagnostic methods for $\mathrm{HPeV} 3$ infection.

Author Contributions: K.G. designed and performed the research, analyzed the data, and wrote the manuscript. Y.Y. performed the research and analyzed the data. K.M., M.N., and H.K. (Hirokazu Kimura) analyzed the data. H.K. (Hajera Khatun), N.N., and T.Y. edited the manuscript. A.R. directed the research, analyzed the data, and wrote the manuscript. All authors have read and agreed to the published version of the manuscript.

Funding: This work was supported in part by the grant for 2019-2020 Strategic Research Promotion (Nos. SK2807) of Yokohama City University to A.R.

Acknowledgments: We thank Naohito Nozaki and Satoko Matsunaga for antibody production and technical assistance, and Masaki Takahashi (Iwate Prefectural Institute of Public Health) for providing regents.

Conflicts of Interest: Y.Y. is a current employee of Kanto Chemical Co., Inc. The remaining authors declare that the research was conducted in the absence of any commercial or financial relationships that could be construed as a potential conflict of interest.

\section{References}

1. Olijve, L.; Jennings, L.; Walls, T. Human Parechovirus: An Increasingly Recognized Cause of Sepsis-Like Illness in Young Infants. Clin. Microbiol. Rev. 2018, 31. [CrossRef] [PubMed]

2. Chuchaona, W.; Khamrin, P.; Yodmeeklin, A.; Saikruang, W.; Kongsricharoern, T.; Ukarapol, N.; Okitsu, S.; Hayakawa, S.; Ushijima, H.; Maneekarn, N. Detection and characterization of a novel human parechovirus genotype in Thailand. Infect. Genet. Evol. 2015, 31, 300-304. [CrossRef] [PubMed]

3. Ito, M.; Yamashita, T.; Tsuzuki, H.; Takeda, N.; Sakae, K. Isolation and identification of a novel human parechovirus. J. Gen. Virol. 2004, 85. [CrossRef] [PubMed]

4. Fernandez-Garcia, M.D.; Simon-Loriere, E.; Kebe, O.; Sakuntabhai, A.; Ndiaye, K. Identification and molecular characterization of the first complete genome sequence of Human Parechovirus type 15. Sci. Rep. 2020, 10. [CrossRef]

5. de Crom, S.C.; Rossen, J.W.; van Furth, A.M.; Obihara, C.C. Enterovirus and parechovirus infection in children: A brief overview. Eur. J. Pediatr. 2016, 175, 1023-1029. [CrossRef]

6. Khatami, A.; McMullan, B.J.; Webber, M.; Stewart, P.; Francis, S.; Timmers, K.J.; Rodas, E.; Druce, J.; Mehta, B.; Sloggett, N.A.; et al. Sepsis-like disease in infants due to human parechovirus type 3 during an outbreak in Australia. Clin. Infect. Dis. 2015, 60, 228-236. [CrossRef]

7. Karelehto, E.; van der Sanden, S.; Geraets, J.A.; Domanska, A.; van der Linden, L.; Hoogendoorn, D.; Koen, G.; van Eijk, H.; Shakeel, S.; Beaumont, T.; et al. Strain-dependent neutralization reveals antigenic variation of human parechovirus 3. Sci. Rep. 2017, 7, 12075. [CrossRef]

8. Selvarangan, R.; Nzabi, M.; Selvaraju, S.B.; Ketter, P.; Carpenter, C.; Harrison, C.J. Human parechovirus 3 causing sepsis-like illness in children from midwestern United States. Pediatr. Infect. Dis. J. 2011, 30, $238-242$. [CrossRef]

9. Boivin, G.; Abed, Y.; Boucher, F.D. Human parechovirus 3 and neonatal infections. Emerg. Infect. Dis. 2005, 11, 103-105. [CrossRef]

10. Levorson, R.E.; Jantausch, B.A.; Wiedermann, B.L.; Spiegel, H.M.; Campos, J.M. Human parechovirus-3 infection: Emerging pathogen in neonatal sepsis. Pediatr. Infect. Dis. J. 2009, 28, 545-547. [CrossRef]

11. Sano, K.; Hamada, H.; Hirose, S.; Sugiura, K.; Harada, S.; Koizumi, M.; Hara, M.; Nishijima, H.; Taira, M.; Ogura, A.; et al. Prevalence and characteristics of human parechovirus and enterovirus infection in febrile infants. Pediatr. Int. 2018, 60, 142-147. [CrossRef] [PubMed] 
12. Harvala, H.; Calvert, J.; Van Nguyen, D.; Clasper, L.; Gadsby, N.; Molyneaux, P.; Templeton, K.; McWilliams Leitch, C.; Simmonds, P. Comparison of diagnostic clinical samples and environmental sampling for enterovirus and parechovirus surveillance in Scotland, 2010 to 2012. Euro. Surveill. 2014, 19. [CrossRef] [PubMed]

13. Britton, P.N.; Dale, R.C.; Nissen, M.D.; Crawford, N.; Elliott, E.; Macartney, K.; Khandaker, G.; Booy, R.; Jones, C.A.; Investigators, P.-A. Parechovirus Encephalitis and Neurodevelopmental Outcomes. Pediatrics 2016, 137, e20152848. [CrossRef] [PubMed]

14. Sedmak, G.; Nix, W.A.; Jentzen, J.; Haupt, T.E.; Davis, J.P.; Bhattacharyya, S.; Pallansch, M.A.; Oberste, M.S. Infant deaths associated with human parechovirus infection in Wisconsin. Clin. Infect. Dis. 2010, 50, 357-361. [CrossRef]

15. Verboon-Maciolek, M.A.; Groenendaal, F.; Hahn, C.D.; Hellmann, J.; van Loon, A.M.; Boivin, G.; de Vries, L.S. Human parechovirus causes encephalitis with white matter injury in neonates. Ann. Neurol. 2008, 64, 266-273. [CrossRef]

16. Vergnano, S.; Kadambari, S.; Whalley, K.; Menson, E.N.; Martinez-Alier, N.; Cooper, M.; Sanchez, E.; Heath, P.T.; Lyall, H. Characteristics and outcomes of human parechovirus infection in infants (2008-2012). Eur. J. Pediatr. 2015, 174, 919-924. [CrossRef]

17. Aizawa, Y.; Izumita, R.; Saitoh, A. Human parechovirus type 3 infection: An emerging infection in neonates and young infants. J. Infect. Chemother. 2017, 23, 419-426. [CrossRef]

18. Mizuta, K.; Yamakawa, T.; Kurokawa, K.; Chikaoka, S.; Shimizu, Y.; Itagaki, T.; Katsushima, F.; Katsushima, Y.; Ito, S.; Aoki, Y.; et al. Epidemic myalgia and myositis associated with human parechovirus type 3 infections occur not only in adults but also in children: Findings in Yamagata, Japan, 2014. Epidemiol. Infect. 2016, 144. [CrossRef]

19. Miyazaki, M.; Hara, K.; Takayoshi, T.; Kawase, T.; Nakagawa, Y.; Arai, T.; Sugimoto, T.; Nishiyama, K.; Gonzalez, G.; Hanaoka, N.; et al. Epidemic Myalgia Associated with Human Parechovirus Type 3 Infection. Intern. Med. 2020, 59. [CrossRef]

20. Romero, J.R.; Selvarangan, R. The human Parechoviruses: An overview. Adv. Pediatr. 2011, 58, 65-85. [CrossRef]

21. Mizuta, K.; Yamakawa, T.; Nagasawa, H.; Itagaki, T.; Katsushima, F.; Katsushima, Y.; Shimizu, Y.; Ito, S.; Aoki, Y.; Ikeda, T.; et al. Epidemic myalgia associated with human parechovirus type 3 infection among adults occurs during an outbreak among children: Findings from Yamagata, Japan, in 2011. J. Clin. Virol. 2013, 58, 188-193. [CrossRef] [PubMed]

22. Nakamura, K.; Saito, K.; Hara, Y.; Aoyagi, T.; Kitakawa, K.; Abe, Y.; Takemura, H.; Ikeda, F.; Kaku, M.; Kanemitsu, K. Severe epidemic myalgia with an elevated level of serum interleukin-6 caused by human parechovirus type 3: A case report and brief review of the literature. BMC Infect. Dis. 2018, 18, 381. [CrossRef]

23. van der Sanden, S.M.; Koopmans, M.P.; van der Avoort, H.G. Detection of human enteroviruses and parechoviruses as part of the national enterovirus surveillance in the Netherlands, 1996-2011. Eur. J. Clin. Microbiol. Infect. Dis. 2013, 32, 1525-1531. [CrossRef]

24. Domanska, A.; Flatt, J.W.; Jukonen, J.J.J.; Geraets, J.A.; Butcher, S.J. A 2.8-Angstrom-Resolution Cryo-Electron Microscopy Structure of Human Parechovirus 3 in Complex with Fab from a Neutralizing Antibody. J. Virol. 2019, 93. [CrossRef] [PubMed]

25. Shakeel, S.; Westerhuis, B.M.; Domanska, A.; Koning, R.I.; Matadeen, R.; Koster, A.J.; Bakker, A.Q.; Beaumont, T.; Wolthers, K.C.; Butcher, S.J. Multiple capsid-stabilizing interactions revealed in a high-resolution structure of an emerging picornavirus causing neonatal sepsis. Nat. Commun. 2016, 7, 11387. [CrossRef] [PubMed]

26. Shakeel, S.; Dykeman, E.C.; White, S.J.; Ora, A.; Cockburn, J.J.B.; Butcher, S.J.; Stockley, P.G.; Twarock, R. Genomic RNA folding mediates assembly of human parechovirus. Nat. Commun. 2017, 8, 5. [CrossRef]

27. Chen, B.C.; Chang, J.T.; Huang, T.S.; Chen, J.J.; Chen, Y.S.; Jan, M.W.; Chang, T.H. Parechovirus A Detection by a Comprehensive Approach in a Clinical Laboratory. Viruses 2018, 10, 711. [CrossRef]

28. Abed, Y.; Wolf, D.; Dagan, R.; Boivin, G. Development of a serological assay based on a synthetic peptide selected from the VP0 capsid protein for detection of human parechoviruses. J. Clin. Microbiol. 2007, 45. [CrossRef] 
29. Yamaoka, Y.; Matsuyama, S.; Fukushi, S.; Matsunaga, S.; Matsushima, Y.; Kuroyama, H.; Kimura, H.; Takeda, M.; Chimuro, T.; Ryo, A. Development of Monoclonal Antibody and Diagnostic Test for Middle East Respiratory Syndrome Coronavirus Using Cell-Free Synthesized Nucleocapsid Antigen. Front. Microbiol. 2016, 7, 509. [CrossRef]

30. Khatun, H.; Yamaoka, Y.; Matsushima, Y.; Matsunaga, S.; Kimura, H.; Ho, J.; Shuda, M.; Ryo, A. Production and characterization of monoclonal antibodies specific for major capsid VP1 protein of trichodysplasia spinulosa-associated polyomavirus. Microbiol. Immunol. 2018, 62, 763-773. [CrossRef]

31. Wildenbeest, J.G.; Benschop, K.S.; Minnaar, R.P.; Bouma-de Jongh, S.; Wolthers, K.C.; Pajkrt, D. Clinical relevance of positive human parechovirus type 1 and 3 PCR in stool samples. Clin. Microbiol. Infect. 2014, 20, O640-O647. [CrossRef] [PubMed]

32. Kadambari, S.; Harvala, H.; Simmonds, P.; Pollard, A.J.; Sadarangani, M. Strategies to improve detection and management of human parechovirus infection in young infants. Lancet Infect. Dis. 2019, 19, e51-e58. [CrossRef]

33. Matsunaga, S.; Kawakami, S.; Matsuo, I.; Okayama, A.; Tsukagoshi, H.; Kudoh, A.; Matsushima, Y.; Shimizu, H.; Okabe, N.; Hirano, H.; et al. Wheat germ cell-free system-based production of hemagglutinin-neuraminidase glycoprotein of human parainfluenza virus type 3 for generation and characterization of monoclonal antibody. Front. Microbiol. 2014, 5, 208. [CrossRef] [PubMed]

34. Nelson, P.N.; Reynolds, G.M.; Waldron, E.E.; Ward, E.; Giannopoulos, K.; Murray, P.G. Monoclonal antibodies. Mol. Pathol. 2000, 53, 111-117. [CrossRef] [PubMed]

35. Sirivichayakul, C.; Limkittikul, K.; Chanthavanich, P.; Yoksan, S.; Ratchatatat, A.; Lim, J.K.; Arunsodsai, W.; Sabchareon, A. Monoclonal antibody-based capture ELISA in the diagnosis of previous dengue infection. Virol. J. 2019, 16, 125. [CrossRef] [PubMed]

36. Pikora, C.; Wittish, C.; Desrosiers, R.C. Identification of two N-linked glycosylation sites within the core of the simian immunodeficiency virus glycoprotein whose removal enhances sensitivity to soluble CD4. J. Virol. 2005, 79, 12575-12583. [CrossRef]

37. Stove, V.; Ramos, P.A.; Wallemacq, P.; Vogeser, M.; Schuetzenmeister, A.; Schmiedel, C.; Shipkova, M. Measurement of sirolimus concentrations in human blood using an automated electrochemiluminescence immunoassay (ECLIA): A multicenter evaluation. Clin. Chem. Lab. Med. 2018, 56, 764-775. [CrossRef]

38. Wada, A.; Sakoda, Y.; Oyamada, T.; Kida, H. Development of a highly sensitive immunochromatographic detection kit for $\mathrm{H} 5$ influenza virus hemagglutinin using silver amplification. J. Virol. Methods 2011, 178, 82-86. [CrossRef]

39. Mishra, M.; Tiwari, S.; Gunaseelan, A.; Li, D.; Hammock, B.D.; Gomes, A.V. Improving the sensitivity of traditional Western blotting via Streptavidin containing Poly-horseradish peroxidase (PolyHRP). Electrophoresis 2019, 40, 1731-1739. [CrossRef] 